\title{
La articulación de la Universidad y la Escuela secundaria. Desafíos educativos ante la agenda $2030^{\prime}$
}

\author{
María Cristina Nin² \\ Universidad Nacional de La Pampa \\ @ [ninmcristina@gmail.com ]
}

DOI: http://dx.doi.org/10.19137/huellas-2019-2318

\section{Introducción}

- 1 contexto actual se caracteriza por la creciente complejidad y la inCterconexión en todos los ámbitos. El desarrollo del conocimiento y las innovaciones científicas y tecnológicas requieren de equipos de trabajo interdisciplinarios y por consiguiente es preciso planificar la educación a partir de un trabajo articulado entre los diferentes ámbitos responsables de su construcción. En este sentido la Agenda 2030 de Desarrollo Sostenible proclamada en 2015 por la Organización de Naciones Unidas (ONU) propone objetivos y metas de carácter integrado e indivisible que contemplan tres dimensiones del desarrollo sostenible: la económica, la social y la ambiental (ONU, 2015).

Los países que integran la ONU se comprometen a invertir, trabajar y difundir el cumplimiento de las metas propuestas. Los gobiernos, el sector privado, la sociedad civil, los sistemas educativos tendrán que contribuir al cumplimiento de las metas correspondientes a cada uno de los objetivos. Es este contexto normativo el que promueve un compromiso articulado entre diversos sectores de la sociedad. Los Estados serían los principales responsables pero el cumplimiento de las metas está en manos de los diferentes agentes. Por lo tanto la articulación de estrategias entre los ámbitos universitarios y otros sectores educativos presenta desafíos de construir

1 Este artículo se enmarca en el Proyecto de Investigación "Geografía y enseñanza (De) construyendo teorías, prácticas, contextos y sujetos". Aprobado por Resolución 042-18-CD-FCHUNLPam.

2 Profesora e Investigadora, Instituto de Geografía, FCH-UNLPam. 
herramientas para trabajar de manera mancomunada ante las problemáticas que presenta la educación.

\section{La Agenda 2030 convoca y desafía a la Universidad}

La Agenda 2030 convoca a diferentes actores sociales para su implementación: públicos, privados, locales, nacionales e internacionales. Entre ellos, las Universidades cumplen un rol destacado debido a que son las responsables de la promoción de la investigación, la creación de innovaciones, la formación de profesionales en distintas áreas del conocimiento. Es decir la Universidad tiene la responsabilidad académica y pedagógica de promover y desarrollar acciones para el cumplimiento de la Agenda 2030. En este sentido la Universidad Nacional de La Pampa en el año 2018 adhiere a "Postulación UNLPam a la Iniciativa impacto académico de Naciones Unidas (UNAI) en a través de la cual La ONU incorpora a la Universidad y ésta se compromete a trabajar para lograr el cumplimiento de los ODS $^{3}$.

Respecto al Objetivo 4 referido a la educación, en el cual la universidad está directamente relacionada, en algunas de sus metas le corresponde de manera clara su responsabilidad. Las metas 4.3, proclaman asegurar el acceso igualitario de todos los hombres y las mujeres a una formación técnica, profesional y superior de calidad, incluida la enseñanza universitaria y la 4.4, aumentar considerablemente el número de jóvenes y adultos que tienen las competencias necesarias, en particular técnicas y profesionales, para acceder al empleo, el trabajo decente y el emprendimiento. Estos postulados nos interpelan como institución formadora y a su vez nos exigen una formación profesional en articulación con el mundo del trabajo (Nin y Shmite, 2019). La igualdad de género, la inclusión de personas con capacidades diferentes y de grupos vulnerables, la formación para el desarrollo sostenible en clave de derechos humanos se explicitan en la metas y ya son ejes de trabajo de las universidades argentinas.

En este contexto generar un espacio que profundice el diálogo y sistematice las acciones que las universidades llevan adelante en relación con la universalización y la mejora calidad de la escuela secundaria es un desafío que plantea demandas y acciones a implementar. Asimismo es importante situar en diálogo las agendas de investigación de las universidades y las políticas sobre la educación secundaria, e identificar recurrencias y vacancias que puedan orientar futuras líneas de investigación sobre educación secundaria a desarrollar en las universidades. De este modo las

3 Resolución $N^{\circ} 441,14$ de noviembre de 2018. Consejo Superior UNLPam. 
problemáticas detectadas en la Escuela secundaria se convierten en asunto de investigación. Entre ellas, las temáticas vinculadas a las trayectorias escolares y las condiciones de escolarización hasta las indagaciones recientes en torno al problema de las transiciones escolares entre niveles, los cambios en las condiciones de trabajo escolar, los cambios curriculares y su apropiación por parte de los diferentes agentes del sistema educativo, los nuevos formatos educativos, entre otros. Es así como, las investigaciones académicas contribuirán a visibilizar problemas que pueden ser insumos para planificar políticas públicas y los problemas detectados por carteras gubernamentales pueden ser objeto de estudio de programas de investigación universitarios.

Debido a la complejidad que presenta el nivel educativo secundario es necesario construir una trama compleja de actores que contribuyan a pensar, planificar y definir estrategias de acción conjunta para resolver los emergentes que presenta. Para ello el trabajo articulado se visualiza como la estrategia más eficiente, coherente y sólida para propiciar la mejora y de ambos niveles.

Entre las acciones que ya están vigentes se encuentran el trabajo de los Proyectos de Extensión Universitaria y las actividades comunitarias ${ }^{4}$ que todos los estudiantes universitarios tienen que desarrollar en el transcurso de su trayectoria. A partir de estas acciones los futuros profesionales establecen vínculos con instituciones del medio donde estudian y se produce un intercambio de saberes imprescindible para su formación profesional.

Por otra parte el Programa NEXOS, vigente desde 2017, promueve una estrategia de integración entre los niveles educativos la cual permite construir diagnósticos compartidos y en consecuencia planes de trabajo en común. Entre otras cuestiones el Programa impulsa estrategias educativas que se focalizan en el último año del nivel secundario y el ingreso a la universidad y espacios de intercambio con referentes nacionales, regionales, jurisdiccionales e institucionales del nivel superior para favorecer la articulación entre el nivel secundario y los distintos subsistemas de educación superior. En síntesis este Programa se constituye en la principal herramienta diseñada para la acción conjunta entre la Universidad y las instituciones de nivel secundario. "El objetivo principal es fortalecer el proceso de inserción de los alumnos en el nivel superior, promover la continuidad de sus estudios, formar para el ejercicio de la ciudadanía y brindar las competencias requeridas por el mundo del trabajo" (Res. N 321/17).

4 En La Universidad Nacional de La Pampa a partir de la Resolución №297-CS-11 del Consejo Superior todos los estudiantes tienen que realizar cuarenta horas de Prácticas Comunitarias antes de finalizar sus estudios, desde el año 2017 son de carácter obligatorio. 
El Programa NEXOS presenta varias líneas de trabajo, una de ellas es la Articulación Universidad-Escuela Secundaria, cuyos principales Ejes de trabajo son el Abordaje de competencias básicas y específicas para el acceso a la educación superior, el Reconocimiento de las diferentes ofertas formativas y las Experiencias orientadas a la formación de vocaciones tempranas. Las líneas de acción a través de las que se implementan son: Tutorías académicas en las escuelas secundarias; Actividades aproximación a la vida universitaria; Producción de material educativo y Estrategias de formación y capacitación permanente.

Otra de las líneas de acción es la Articulación hacia el interior del sistema de educación superior, a partir de este subprograma se pretende promover la planificación y articulación del nivel para fortalecer las necesidades de formación. Promueve la articulación entre el sistema universitario y las instituciones de formación superior no universitaria tanto docente como técnica. Las líneas de acción son; Creación de redes de comunicación entre distintos actores nacionales, regionales y locales; Compilación de normativa vigente para ambos subsistemas y diseño de nuevos instrumentos para mejorar su organización; Reactivación de estrategias de articulación entre las Universidades y los Institutos de Educación Superior ya implementadas a nivel local, jurisdiccional y regional y la Elaboración de convenios entre instituciones, jurisdicciones $\mathrm{y} / \mathrm{o}$ regiones para favorecer el trayecto formativo de los estudiantes.

La sistematización de encuentros con un espacio común de debate y reflexión que involucre a las universidades y a los responsables de los diseños y puesta en marcha de políticas públicas permitirá profundizar acciones y políticas públicas que caminen hacia la democratización y cobertura de todos los niveles educativos.

\section{Reflexiones finales}

La agenda de políticas educativas que se promueve desde el Ministerio de Educación Nacional, la Secretaría de Políticas Universitarias, y que las Universidades y los Ministerios de Educación Provinciales efectivizan con acciones concretas intenta resolver problemáticas y afrontar diagnósticos del sistema educativo. La propuesta supera el aislamiento institucional, por el contrario fortalece la articulación entre la Universidad y el Nivel Secundario. La obligatoriedad del nivel secundario es un proceso que requiere de la construcción permanente e interpela al Estado y a todas las instituciones educativas a pensar la educación en clave de derecho social y a los estudiantes como sujetos de derecho. El acceso a la Universidad se convierte 
en derecho social desde la Ley de Educación Nacional (2006) y su consecuente obligatoriedad de la educación secundaria.

La construcción de una agenda conjunta que genere acuerdos sustentados en base a las necesidades detectadas en cada región, propios de cada nivel y que focalicen las miradas en el trabajo articulado para el pasaje de los niveles con el respeto de las especificidades de cada uno, contribuirá a construir una educación más democrática. La Universidad pública argentina tiene el compromiso ético de generación conocimientos científicos que respondan a las demandas de la sociedad.

En la agenda pública se instaló el debate y las acciones, dependerá de los diferentes actores involucrados favorecer la consolidación de una trama justa y académicamente responsable en pos de la construcción de futuros con derechos sociales para todas y todos.

\section{Referencias bibliográficas}

Alonso. J. A. (2018). Universidad: Investigación e innovación al servicio de la Agenda 2030. En: El papel de la Universidad Iberoamericana en la Agenda 2030. (2018). Seminario de la SEGIB. Salamanca. Febrero 2018. Disponible en http://www.ocud.es/agenda2030/ es/files/doc39/documento-conferencia-universidades-segib.pdf.

Consejo Federal de Educación. (2017). Resolución $\mathrm{N}^{\circ}$ 321/17. "Programa Nexos: Articulación y Cooperación Educativa". Recuperado de: https://www.argentina.gob.ar/sites/default/ files/resolucion_consejo_federal_de_educacion_res_cfe_ndeg321-17.. pdf

Consejo Federal de Educación. (2017). Anexo Resolución No 321/17. "Programa Nexos: Articulación y Cooperación Educativa". Disponible en: https://www.argentina.gob. $\mathrm{ar} / \mathrm{sites} /$ default/files/resolucion_consejo_federal_de_educacion_res_cfe_ndeg $321-\overline{17}$. anexo_i.pdf

Consejo Nacional de Coordinación de Políticas Sociales (2017). Estado de Avance de la implementación de la Agenda 2030 en Argentina. Buenos Aires. Disponible en : http://www. onu.org.ar/stuff/ODS_Estado_Avance.pdf.

Ministerio de Educación. Provincia de La Pampa. 2030. Escenarios de la Educación en la Provincia de La Pampa. Tensiones, desafíos y acuerdos. Disponible en : https://sitio.lapampa.edu.ar/repositorio/programas_proyectos/ educacion_2030/2030.pdf

Ministerio de Cultura, Ciencia y Tecnología. (s/f). Programa Nexos. Disponible en https:/www. argentina.gob.ar/educacion/universidades/ programa-nexos

Nin, M.C. y Shmite. S. M. (2019). "Objetivos de Desarrollo Sostenible. Desafíos de la Universidad ante la Agenda 2030". En Dillon, B, Nin, M.C. y Pombo, D (Compiladoras). Repensar las geografias para construir saberes en contextos dinámicos. pp. 167-175. Santa Rosa: EdULPam.

Nin, M. C. y Shmite S. M. (2018). "Naciones Unidas y Los Objetivos de Desarrollo Sostenible. Aportes desde la Enseñanza de la Geografía". En Lell, H. (Comp.) Actas del II Congreso Internacional Instituciones e interdisciplina: alcances jurídicos, económicos y epistemológicos. Pp. 252-263. Universidad Nacional de La Pampa, Santa Rosa.

Objetivos de Desarrollo del Milenio - ODM. INFORME 2015. ONU, Nueva York. Disponible en http://www.un.org/es/millenniumgoals/ pdf/2015/mdg-report-2015_spanish.pdf

Objetivos de Desarrollo Sostenible - ODS (2015). ONU, Nueva York. Disponible en: http:// 
www.un.org/sustainabledevelopment/es/ objetivos-de-desarrollo-sostenible/

ONU (2015). Proyecto de documento final de la cumbre de las Naciones Unidas para la aprobación de la agenda para el desarrollo después de 2015. Documento A/69/L.85. 12 de agosto de 2015. ONU. Disponible en http://200.23.8.225/odm/Doctos/TNM_2030. pdf 